\title{
On Lower Bounds and Uniqueness for Solutions of the Navier-Stokes Equations
}

\section{HAJIMU OGAWA*}

\author{
Communicated by J. B. Diaz
}

1. Introduction. Consider the motion of a viscous incompressible fluid in a domain $\Omega$ in $E^{m}, m \geqq 2$, subject to the condition that the fluid velocity $u$ vanishes on the boundary of $\Omega$. In a recent paper, Edmunds [5] studied the asymptotic behavior of $u$ as the time $t$ tends to infinity. For a bounded domain, he showed that if the first order space derivatives of $u$ are uniformly of order $O\left(e^{-\mu t}\right)$ as $t \rightarrow \infty$ for every $\mu>0$, then $u$ vanishes identically. He also proved, in case $\Omega$ is the complement of a bounded subset of $E^{m}$, if $u, \partial u / \partial t$ and grad $u$ are uniformly $O\left(e^{-\mu t}\right)$ as $t \rightarrow \infty$ for every $\mu>0$, and $u$ and the pressure $p$ satisfy certain growth conditions in $x$ and $t$, then $u$ is identically zero.

The object of this paper is to establish lower bounds for the velocity $u$ from which will follow asymptotic results of the type just described. This is done in Section 2 for bounded domains and in Section 3 for unbounded domains. Although the spatial growth conditions imposed in the case of unbounded domains are not entirely comparable with those of Edmunds, for both bounded and unbounded domains the result is obtained with only the $L_{2}$ norm of $u$ being required to decay exponentially in time.

As a further application, in Section 4 we shall show that the forward and backward uniqueness theorems of Serrin [9] for bounded domains are easy consequences of the bounds of Section 2. For unbounded domains, Graffi [6] established a forward uniqueness theorem under the assumptions that $u$ and its first partial derivatives are uniformly bounded on each finite time interval and that for some constant $p_{0}, p-p_{0}=O\left(|x|^{-1}\right)$ as $|x|^{2}=\sum x_{i}^{2} \rightarrow \infty$, uniformly on finite time intervals. A backward uniqueness theorem was found by Edmunds [4] under somewhat stronger conditions, except that the condition on the pressure was weakened to $p-p_{0}=O\left(|x|^{-1 / 2-\epsilon}\right)$ for some $\epsilon>0$. Uniqueness theorems of this kind also follow from the results of Section 3. However, because of spatial growth restrictions, the theorems are not as strong as those in [4] and [6].

The methods are elementary and are based on the convexity type inequalities which are satisfied by solutions of certain differential inequalities in Hilbert

* This work was supported in part by the National Science Foundation. 
space and parabolic differential inequalities; $c f$. Cohen and Lees [3], Agmon and Nirenberg [1], [2] and Ogawa [7], [8].

2. Lower bounds on bounded domains. Let $\Omega$ be a bounded open subset of $E^{m}$ with boundary $\partial \Omega$ and suppose Green's theorem is valid in $\Omega$. It will be convenient to introduce the following notation. For vector-valued functions $g=\left(g_{1}, \cdots, g_{m}\right), h=\left(h_{1}, \cdots, h_{m}\right)$ in $C^{1}(\bar{\Omega} \times[0, T)), 0<T \leqq \infty$, set

$$
\begin{aligned}
(g(t), h(t)) & =\int_{\Omega} g(x, t) \cdot h(x, t) d x, \quad|g(t)|^{2}=(g(t), g(t)), \\
\|g(t)\|^{2} & =\int_{\Omega}|\operatorname{grad} g(x, t)|^{2} d x=\int_{\Omega} \sum_{i, j=1}^{m}\left(\frac{\partial g_{i}}{\partial x_{j}}\right)^{2} d x .
\end{aligned}
$$

For brevity we shall also write $|g|=|g(t)|$. In order that $|g|$ not be confused with the Euclidean norm of the vector $g$, we shall always indicate the arguments in the latter; i.e.,

$$
|g(x, t)|^{2}=\sum_{i=1}^{m} g_{i}^{2}(x, t)
$$

If $u=\left(u_{1}, \cdots, u_{m}\right)$ denotes the velocity, $p$ the pressure and $f=\left(f_{1}, \cdots, f_{m}\right)$ the external force, the Navier-Stokes equations are given by

$$
\begin{aligned}
\frac{\partial u}{\partial t}-\Delta u+u \cdot \operatorname{grad} u & =-\operatorname{grad} p+f, \\
\operatorname{div} u & =0 .
\end{aligned}
$$

We shall assume that $f_{\varepsilon} C(\bar{\Omega} \times[0, T))$ and satisfies

$$
|f(t)| \leqq F(t)\|u(t)\|
$$

for some measurable function $F$. We also set

$$
U(t)=\sup _{x \in \Omega}|u(x, t)| .
$$

Theorem 1. Let $\Omega$ be a bounded domain. Let $u \varepsilon C^{2}(\bar{\Omega} \times[0, T))$ and $p$ $\varepsilon C^{1}(\bar{\Omega} \times[0, T))$ be a solution of the Navier-Stokes equations with $u=0$ on $\partial \Omega \times$ $[0, T)$. Suppose $U$ and $F$ are in $L_{2}[0, T)$.

(i) If $|u(\mathrm{t})|>0$ for $0 \leqq t_{0} \leqq t<T$, then

$$
|u(t)| \geqq K\left|u\left(t_{0}\right)\right| e^{-\lambda\left(t-t_{0}\right)}, \quad t_{0} \leqq t<T,
$$

where $K$ and $\lambda$ are positive constants depending on $u\left(t_{0}\right)$ and the $L_{2}$ norms of $U$ and $F$.

(ii) Let $T=\infty$. If $|u(t)|=O\left(e^{-\mu t}\right)$ as $t \rightarrow \infty$ for each $\mu>0$, then $u \equiv 0$.

Proof. We first consider

$$
\begin{aligned}
\frac{d}{d t}|u|^{2} & =2\left(u, \frac{\partial u}{\partial t}\right) \\
& =2(u, \Delta u-u \cdot \operatorname{grad} u-\operatorname{grad} p+f) .
\end{aligned}
$$


Using Green's theorem and the fact that $u$ has zero divergence, we find

$$
(u, u \cdot \operatorname{grad} u)=0, \quad(u, \operatorname{grad} p)=0 .
$$

Since $u$ vanishes on the boundary, it follows that

$$
\frac{d}{d t}|u|^{2}=-2\|u\|^{2}+2(u, f) .
$$

Similarly

$$
\begin{aligned}
\|u\|^{2} & =-(u, \Delta u)=-\left(u, \frac{\partial u}{\partial t}+u \cdot \operatorname{grad} u-f\right), \\
\frac{d}{d t}\|u\|^{2} & =2\left(\operatorname{grad} \frac{\partial u}{\partial t}, \operatorname{grad} u\right)=-2\left(\frac{\partial u}{\partial t}, \Delta u\right) \\
& =-2\left(\frac{\partial u}{\partial t}, \frac{\partial u}{\partial t}+u \cdot \operatorname{grad} u-f\right) .
\end{aligned}
$$

Letting $Q(t)=\|u(t)\|^{2} /|u(t)|^{2}$ we obtain for $t_{0} \leqq t<T$

$$
\begin{array}{r}
\frac{d Q}{d t}=-\frac{2}{|u|^{4}}\left[|u|^{2}\left(\frac{\partial u}{\partial t}, \frac{\partial u}{\partial t}+u \cdot \operatorname{grad} u-f\right)-\left(u, \frac{\partial u}{\partial t}\right)\left(u, \frac{\partial u}{\partial t}+u \cdot \operatorname{grad} u-f\right)\right] \\
=-\frac{2}{|u|^{4}}\left[|u|^{2}\left|\frac{\partial u}{\partial t}+\frac{1}{2}(u \cdot \operatorname{grad} u-f)\right|^{2}-\frac{1}{4}|u|^{2}|u \cdot \operatorname{grad} u-f|^{2}\right. \\
\left.-\left(u, \frac{\partial u}{\partial t}+\frac{1}{2}(u \cdot \operatorname{grad} u-f)\right)^{2}+\frac{1}{4}(u, u \cdot \operatorname{grad} u-f)^{2}\right] .
\end{array}
$$

Then by Schwarz's inequality,

$$
\frac{d Q}{d t} \leqq \frac{1}{2} \frac{|u \cdot \operatorname{grad} u-f|^{2}}{|u|^{2}} \leqq \frac{|u \cdot \operatorname{grad} u|^{2}}{|u|^{2}}+\frac{|f|^{2}}{|u|^{2}} .
$$

Making use of (2) and (3), we obtain the differential inequality

$$
\frac{d Q}{d t} \leqq\left(U^{2}+F^{2}\right) Q
$$

which can easily be integrated to give

$$
Q(t) \leqq Q\left(t_{0}\right) \exp \left[\int_{t_{0}}^{t}\left(U^{2}+F^{2}\right) d s\right], \quad t_{0} \leqq t<T .
$$

From the identity (5) we find that

$$
\frac{d}{d t} \log |u|=-Q+\frac{(u, f)}{|u|^{2}}
$$

and thus

$$
\frac{d}{d t} \log |u| \geqq-Q-F Q^{1 / 2} \geqq-2 Q-\frac{1}{4} F^{2}
$$


Inserting (6) into this inequality and integrating, we obtain

(7) $|u(t)| \geqq\left|u\left(t_{0}\right)\right| \exp \left\{-2 Q\left(t_{0}\right) \int_{t_{0}}^{t} \exp \left[\int_{t_{0}}^{\eta}\left(U^{2}+F^{2}\right) d s\right] d \eta-\frac{1}{4} \int_{t_{0}}^{t_{0}} F^{2}(\eta) d \eta\right\}$.

Since $U$ and $F$ are square integrable by hypothesis, the lower bound (4) is an immediate consequence of (7).

It remains to establish (ii). If $u \neq \equiv 0$ on $\Omega \times[0, \infty)$, then for some $t_{0} \geqq 0$, $\left|u\left(t_{0}\right)\right|>0$. By continuity it follows that $|u(t)|>0$ on some interval $\left[t_{0}, T\right)$. Let $\left[t_{0}, T\right)$ be the largest interval with this property. If $T$ is finite, then the lower bound (4) implies $|u(T)|>0$, a contradiction. Hence $|u(t)|>0$ on $\left[t_{0}, \infty\right)$ and consequently (4) is valid for all $t \geqq t_{0}$. However, by hypothesis $e^{\lambda t}|u(t)| \rightarrow 0$ as $t \rightarrow \infty$, which when applied to (4) leads to the contradiction $\left|u\left(t_{0}\right)\right|=0$.

3. Lower bounds on unbounded domains. We now suppose $\Omega$ is the complement of a bounded closed subset of $E^{m}$. Let $B(r)$ denote the open ball $\{x:|x|<r\}$ in $E^{m}$, and for $r$ so large that $S(r)=\partial B(r)$ is contained in $\Omega$, we define $\Omega(r)=\Omega \cap B(r)$. For vector-valued functions $g$ and $h$ in $C^{\mathbf{1}}(\bar{\Omega} \times[0, T))$ we take $(g(t), h(t)),|g(t)|$ and $\|g(t)\|$ as in Section 2 (provided the integrals exist) and set

$$
\begin{gathered}
(g(t), h(t))_{r}=\int_{\Omega(r)} g(x, t) \cdot h(x, t) d x, \quad|g(t)|_{r}^{2}=(g(t), g(t))_{r}, \\
\|g(t)\|_{r}^{2}=\int_{\Omega(r)}|\operatorname{grad} g(x, t)|^{2} d x, \\
(g(t), h(t))_{S(r)}=\int_{S(r)} g(x, t) \cdot h(x, t) d S, \quad|g(t)|_{S(r)}^{2}=(g(t), g(t))_{S(r)}, \\
\|g(t)\|_{S(r)}^{2}=\int_{S(r)}|\operatorname{grad} g(x, t)|^{2} d S .
\end{gathered}
$$

In the Navier-Stokes equations (2) we now assume that the external force satisfies

$$
|f(t)|_{r} \leqq F(t)\|u(t)\|_{r}
$$

for some measurable function $F$. We shall restrict our considerations to the following class of solutions of (2).

Definition. A solution $u, p$ of the Navier-Stokes equations on $\Omega \times[0, T)$ will be said to be of class $S$ if:

(a) $|u(t)|<\infty$ for each $t \varepsilon[0, T)$ and $|u(t)|_{r} \rightarrow|u(t)|$ as $r \rightarrow \infty$, uniformly on closed subintervals of $[0, T)$.

(b) $\|u(t)\|<\infty$ for each $t \varepsilon[0, T)$.

(c) $|u|_{S(r)}=O\left(r^{-1 / 2}\right),|\partial u / \partial t|_{r}=O(1)$ and $|\partial u / \partial t|_{s(r)}=O\left(r^{-1 / 2}\right)$ as $r \rightarrow \infty$, uniformly on closed subintervals of $[0, T)$.

(d) $\|u\|_{s(r)}=o\left(r^{1 / 2}\right),|p|_{s(r)}=o\left(r^{1 / 2}\right)$ as $r \rightarrow \infty$, uniformly on closed subintervals of $[0, T)$. 
The condition (a), which is required to assure the continuity of $|u(t)|$, does not correspond to any of Edmunds' assumptions, while (b) was one of his restrictions. The properties (c) together with the exponential decay of $|u(t)|$ and the condition $U \in L_{2}[0, T)$ replace his requirements that $u$ and its first partial derivatives be uniformly bounded for each $t$ and that the bounds decay exponentially with $t$. Finally, while Edmunds' conditions

$$
\|u(t)\|_{S(r)} \leqq B(t) r^{1 / 2-\epsilon}, p(x, t) \leqq A(t) r^{1-m / 2-\epsilon},
$$

for bounded functions $A(t)$ and $B(t)$ and some $\epsilon>0$, are almost equivalent to (d), it was also assumed that $A$ and $B$ decay exponentially.

Theorem 2. Let $\Omega$ be the complement of a bounded closed set. Let $u \varepsilon C^{2}(\bar{\Omega} \times[0, T))$ and $p \varepsilon C^{1}(\bar{\Omega} \times[0, T))$ be a solution of the Navier-Stokes equations of class $S$ with $u=0$ on $\partial \Omega \times[0, T)$. Suppose $U$ and $F$ are in $L_{2}[0, T)$.

(i) If $|u(t)|>0$ for $0 \leqq t_{0} \leqq t<T$, then a lower bound of the form (4) is valid.

(ii) Let $T=\infty$. If $|u(t)|=O\left(e^{-\mu t}\right)$ as $t \rightarrow \infty$ for each $\mu>0$, then $u \equiv 0$.

Proof. For each domain $\Omega(r)$ we proceed as in the proof of Theorem 1, except that we must now take into account the fact that $u$ does not necessarily vanish on $S(r)$. Thus

$$
\frac{d}{d t}|u|_{r}^{2}=-2\|u\|_{r}^{2}-2(u, u \cdot \operatorname{grad} u-f)_{r}+2(u, \operatorname{grad} u \cdot n-p n)_{s(r)},
$$

where $n=\left(n_{1}, \cdots, n_{m}\right)$ is the outward normal to $S(r)$. We next see that

$$
\|u\|_{r}^{2}=-\left(u, \frac{\partial u}{\partial t}+u \cdot \operatorname{grad} u-f\right)_{r}+(u, \operatorname{grad} u \cdot n-p n)_{S(r)}
$$

and

$$
\frac{d}{d t}\|u\|_{r}^{2}=-2\left(\frac{\partial u}{\partial t}, \frac{\partial u}{\partial t}+u \cdot \operatorname{grad} u-f\right)_{r}+2\left(\frac{\partial u}{\partial t}, \operatorname{grad} u \cdot n-p n\right)_{S(r)} .
$$

From (9), (10) and (11) it follows that for $t_{0} \leqq t<T$,

$$
\begin{aligned}
\frac{d Q_{r}}{d t}=- & \frac{2}{|u|_{r}^{2}}\left[\left(\frac{\partial u}{\partial t}, \frac{\partial u}{\partial t}+u \cdot \operatorname{grad} u-f\right)_{r}-\left(\frac{\partial u}{\partial t}, \operatorname{grad} u \cdot n-p n\right)_{s(r)}\right] \\
& +\frac{2}{|u|_{r}^{4}}\left(u, \frac{\partial u}{\partial t}\right)_{r}\left[\left(u, \frac{\partial u}{\partial t}+u \cdot \operatorname{grad} u-f\right)_{r}-(u, \operatorname{grad} u \cdot n-p n)_{S(r)}\right],
\end{aligned}
$$

where we have set $Q_{r}(t)=\|u(t)\|_{r}^{2} /|u(t)|_{r}^{2}$. Repeating the argument in the proof of Theorem 1, we find

$$
\begin{aligned}
& \frac{d Q_{r}}{d t} \leqq\left(U^{2}+F^{2}\right) Q_{r}+\frac{2}{|u|_{r}^{3}}\left[|u|_{r}\left|\frac{\partial u}{\partial t}\right|_{S(r)}+|u|_{S(r)}\left|\frac{\partial u}{\partial t}\right|_{r}\right] \\
& \cdot\left[\sum_{i=1}^{m}\left|\operatorname{grad} u_{i}\right|_{S(r)}+|p|_{S(r)}\right]
\end{aligned}
$$


for $t_{0} \leqq t<T$. Using the condition (a) of class $S$, we see that given any $t_{1}$ with $t_{0}<t_{1}<T$, there exist positive constants $r_{0}$ and $C$ such that $|u(t)|_{r} \geqq C$ for $t_{0} \leqq t \leqq t_{1}$ and $r \geqq r_{0}$. By virtue of the properties (c) and (d) of the class $S$, it follows that

$$
\frac{d Q_{r}}{d t} \leqq\left(U^{2}+F^{2}\right) Q_{r}+\epsilon_{1}\left(r, t_{1}\right), \quad t_{0} \leqq t \leqq t_{1},
$$

where $\epsilon_{1}\left(r, t_{1}\right) \rightarrow 0$ as $r \rightarrow \infty$. The solution of (12) is easily found to be

$Q_{r}(t) \leqq Q_{r}\left(t_{0}\right) \exp \left[\int_{t_{0}}^{t}\left(U^{2}+F^{2}\right) d s\right]+\epsilon_{1}\left(r, t_{1}\right) \int_{t_{0}}^{t_{1}} \exp \left[\int_{\eta}^{t}\left(U^{2}+F^{2}\right) d s\right] d \eta$,

for $t_{0} \leqq t \leqq t_{1}$. Letting $r \rightarrow \infty$, we see that the estimate (6) is valid in this case and since there is no dependence on $t_{1},(6)$ holds for $t_{0} \leqq t<T$. In a similar manner, from the identity (9) it can be seen that for $t_{0} \leqq t \leqq t_{1}$

$$
\frac{d}{d t} \log |u|_{r}^{2} \geqq-2 Q_{r}-\frac{1}{2}\left(U^{2}+F^{2}\right)-\epsilon_{2}\left(r, t_{1}\right),
$$

where $\epsilon_{2}\left(r, t_{1}\right) \rightarrow 0$ as $r \rightarrow \infty$. Integrating, passing to the limit and making use of (6), we find that a lower bound similar to (7) holds for $t_{0} \leqq t<T$. Since $\left\|u\left(t_{0}\right)\right\|<\infty$ and $U$ and $F$ are in $L_{2}[0, T)$, the first part of Theorem 2 is proved. The proof of (ii) is identical with the proof of the second part of Theorem 1 .

4. Uniqueness theorems. As a further application of the lower bounds of Theorems 1 and 2, we next study the uniqueness of solutions of the initial and final value problems for the Navier-Stokes equations. We first consider the case of a bounded domain.

Theorem 3. Let $\Omega$ be a bounded domain. Let $u^{(i)} \varepsilon C^{2}(\bar{\Omega} \times[0, T))$ and $p^{(i)} \varepsilon$ $C^{1}(\bar{\Omega} \times[0, T)), i=1,2$, be two solutions of the Navier-Stokes equations with $u^{(1)}=u^{(2)}$ on $\partial \Omega \times[0, T)$. If $u^{(1)}\left(x, t_{0}\right)=u^{(2)}\left(x, t_{0}\right)$ for all $x \varepsilon \Omega$ and some $t_{0}$, $0<t_{0}<T$, then $u^{(1)} \equiv u^{(2)}$.

Proof. Letting $u=u^{(1)}-u^{(2)}$ and $p=p^{(1)}-p^{(2)}$, we see that

$$
\begin{gathered}
\frac{\partial u}{\partial t}-\Delta u+u^{(1)} \cdot \operatorname{grad} u+\operatorname{grad} u^{(2)} \cdot u=-\operatorname{grad} p, \\
\operatorname{div} u=0,
\end{gathered}
$$

and $u$ vanishes on the boundary. By the techniques used in proving Theorem 1 , we find that if $|u|>0$,

$$
\frac{d Q}{d t} \leqq \frac{1}{2} \frac{\left|u^{(1)} \cdot \operatorname{grad} u+\operatorname{grad} u^{(2)} \cdot u\right|^{2}}{|u|^{2}}
$$

Setting

$$
U_{i}(t)=\max \left[\sup _{x \varepsilon \Omega}\left|u^{(i)}(x, t)\right|, \sup _{x \varepsilon \Omega}\left|\operatorname{grad} u^{(i)}(x, t)\right|\right]
$$


we then have

$$
\frac{d Q}{d t} \leqq U_{1}^{2} Q+U_{2}^{2}
$$

Also

$$
\frac{d}{d t} \log |u|=-Q-\frac{\left(u, u^{(1)} \cdot \operatorname{grad} u+\operatorname{grad} u^{(2)} \cdot u\right)}{|u|^{2}}
$$

from which it follows that

$$
\frac{d}{d t} \log |u| \geqq-Q-U_{1} Q^{1 / 2}-U_{2} .
$$

We are given $\left|u\left(t_{0}\right)\right|=0$. Suppose there exists a number $t_{1}$ with $0 \leqq t_{1}<t_{0}$ such that $\left|u\left(t_{1}\right)\right|>0$. Clearly, we may assume $|u(t)|>0$ for $t_{1} \leqq t<t_{0}$. Integrating (13) from $t_{1}$ to $t$, we find that $Q(t)$ is bounded by a constant depending on $u\left(t_{1}\right)$. Applying this fact to (15), we obtain

$$
|u(t)| \geqq\left|u\left(t_{1}\right)\right| e^{-A\left(t-t_{1}\right)},
$$

where $A$ is a constant depending on $t_{1}$. Because of the continuity of $|u(t)|$, we conclude that $\left|u\left(t_{0}\right)\right|>0$, which is a contradiction. Hence $u \equiv 0$ on $\left[0, t_{0}\right)$.

The fact that $u$ vanishes identically on $\left(t_{0}, T\right)$ is an easy consequence of the identity (14), since

$$
\frac{d}{d t} \log |u| \leqq-Q+U_{1} Q^{1 / 2}+U_{2} \leqq \frac{1}{4} U_{1}^{2}+U_{2}^{2} .
$$

Now suppose $|u(t)|>0$ for $t_{0}<t \leqq t_{2}$. Then

$$
|u(t)| \geqq\left|u\left(t_{2}\right)\right| e^{-B\left(t_{2}-t\right)}
$$

for some constant $B$. But this gives rise to the contradiction $\left|u\left(t_{0}\right)\right|>0$.

The uniqueness in the case of an unbounded domain is established by a straightforward combination of the proofs of Theorems 2 and 3. Because of the restrictions of the class $S$, the result is weaker than the theorems of Graffi [6] and Edmunds [4].

Theorem 4. Let $\Omega$ be the complement of a bounded closed set. Let $u^{(i)} C^{2}(\bar{\Omega} \times$ $[0, T))$ and $p_{\varepsilon}^{(i)} C^{1}(\bar{\Omega} \times[0, T)), i=1,2$, be two solutions of the Navier-Stokes equations of class $S$ such that $u^{(1)}=u^{(2)}$ on $\partial \Omega \times[0, T)$. Suppose the functions $U_{1}$ and $U_{2}$ are in $L_{2}$ on each closed subinterval of $[0, T)$. If $u^{(1)}\left(x, t_{0}\right)=u^{(2)}\left(x, t_{0}\right)$ for all $x \varepsilon \Omega$ and some $t_{0}, 0<t_{0}<T$, then $u^{(1)} \equiv u^{(2)}$.

\section{REFERENCES}

[1] S. Agmon \& L. Nirenberg, Properties of solutions of ordinary differential equations in Banach space, Comm. Pure Appl. Math., 16 (1963) 121-239.

[2] S. Agmon \& L. Nirenberg, Lower bounds and uniqueness theorems for solutions of differential equations in a Hilbert space, Comm. Pure Appl. Math., 20 (1967) 207-229. 
[3] P. J. Cohen \& M. LeEs, Asymptotic decay of solutions of differential inequalities, Pacific .J. Math., 11 (1961) 1235-1249.

[4] D. E. Edmunds, On the uniqueness of viscous flows, Arch. Rat. Mech. Anal., 14 (1963) 171-176.

[5] D. E. Edmunds, Asymptotic behavior of solutions of the Navier-Stokes equations, Arch. Rat. Mech. Anal., 22 (1966) 15-21.

[6] D. Grafri, Sul teorema di unicità nella dinamica dei fluidi, Ann. Mat. Pura Appl., (IV) 50 (1960) 379-387.

[7] H. Ogawa, Lower bounds for solutions of differential inequalities in Hilbert space, Proc. Amer. Math. Soc., 16 (1965) 1241-1243.

[8] H. Ogawa, Lower bounds for solutions of parabolic differential inequalities, Canad. $J$. Math., 19 (1967) 667-672.

[9] J. Serrin, The Initial Value Problem for the Navier-Stokes Equations, Nonlinear Problems, University of Wisconsin Press, Madison, Wisconsin, 1963, pp. 69-98.

Courant Institute of Mathematical Sciences

New York, New York and

University of California

Riverside, California

Date communicated: February 12, 1968 